\title{
Traduire
}

Revue française de la traduction

$221 \mid 2009$

Voies de l'interprétation

\section{Interprètes en langue des signes : autour de l'intervention de Marie-Thérèse L'Huillier à la Journée Mondiale de la Traduction 2008}

Françoise Wirth

\author{
(2) OpenEdition \\ Journals \\ Édition électronique \\ URL : http://journals.openedition.org/traduire/344 \\ DOI : $10.4000 /$ traduire.344 \\ ISSN : 2272-9992 \\ Éditeur \\ Société française des traducteurs
}

Édition imprimée

Date de publication : 15 décembre 2009

Pagination : 33-38

ISSN : 0395-773X

\section{Référence électronique}

Françoise Wirth, «Interprètes en langue des signes : autour de l'intervention de Marie-Thérèse

L'Huillier à la Journée Mondiale de la Traduction 2008 », Traduire [En ligne], 221 | 2009, mis en ligne le

12 novembre 2013, consulté le 10 décembre 2020. URL : http://journals.openedition.org/traduire/344

; DOI : https://doi.org/10.4000/traduire.344 


\section{Interprètes en langue des signes : autour de l'intervention de Marie-Thérèse L'Huillier à la Journée Mondiale de la Traduction 2008}

\section{Françoise Wirth}

Peut-être avez-vous eu la chance d'assister à la Journée mondiale de la traduction 2008 ? Dans ce cas, nul doute que vous aurez été passionnés par la présentation en langue des signes de Mme Marie-Thérèse L'Huillier et épatés par la performance de Guylaine Paris et Jérémie Segouat, interprètes en langue des signes. Nous tenterons ici de reprendre l'essentiel de cette intervention, joliment intitulée "Langues des signes : des concepts plein les yeux ".

Pour pallier leur carence auditive, les sourds ont recours aux autres moyens à leur disposition : les yeux, pour une perception immédiate du monde alentour, le corps et les mains pour s'exprimer sous forme de signes (unités gestuelles).

La Langue des signes française (LSF) est donc une langue visuelle et gestuelle utilisée par la communauté des sourds pour établir une communication directe qui ne transite pas par la voix. Langue minoritaire dont la pratique n'est associée à aucun territoire, elle fait partie de l'identité de la culture sourde. En France, on évalue le nombre des sourds à cinq millions d'individus et à 800000 le nombre des sourds " signants".

Dès lors se pose la question des différences de représentation entre utilisateurs de la langue des signes et utilisateurs d'une langue vocalisée.

Les perspectives perceptivo-pratiques ne sont pas les mêmes, notamment du fait de l'iconicisation $^{(1)}$. Le schéma suivant illustre les différences de perspectives entre langue des signes et langues vocalisées :

(1) Cuxac (2000) appelle iconicité des signes « le lien de ressemblance entre la forme des gestes utilisés et ce à quoi ils réfèrent ". 

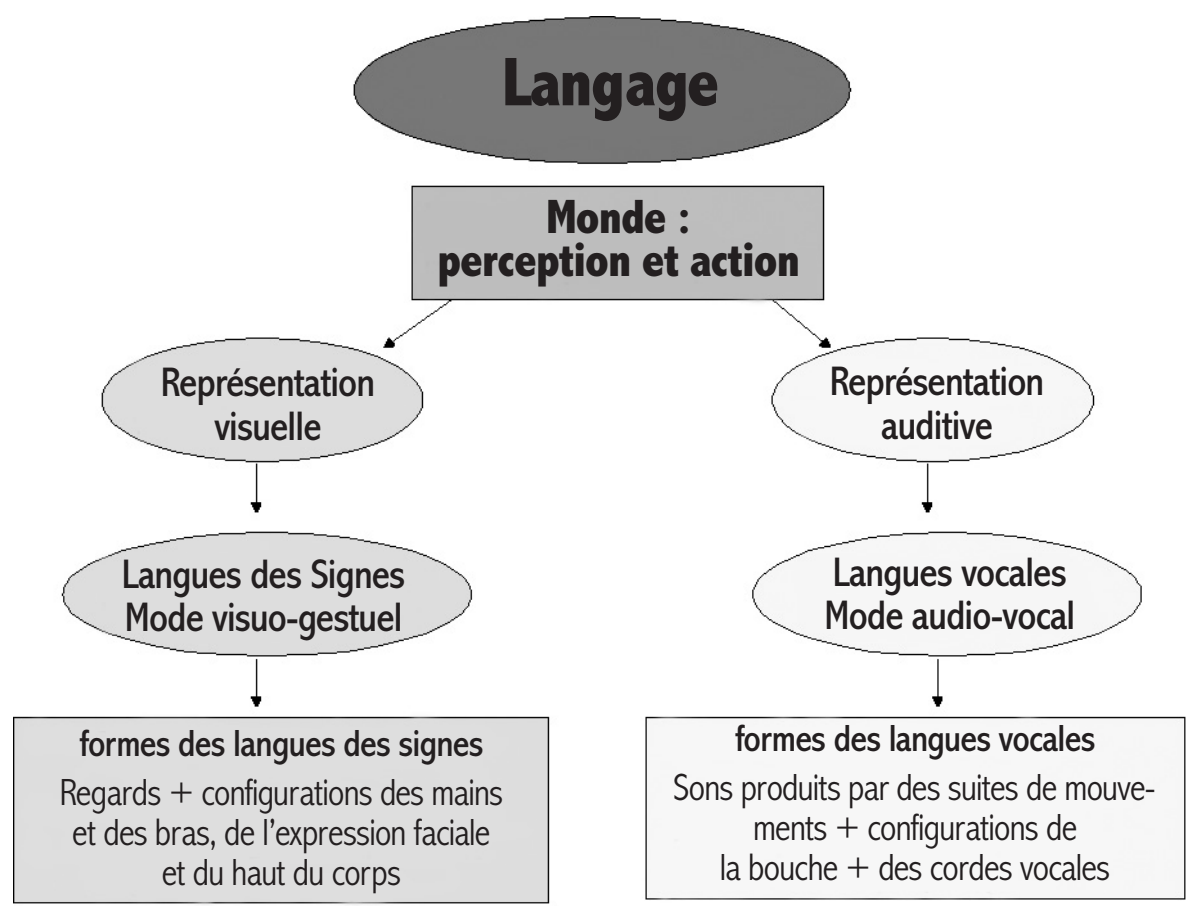

Schéma extrait de la présentation de Mme Marie-Thérèse L'Huillier à la JMT 2008

Contrairement à celle de la langue française, la grammaire de la LSF n'est pas linéaire mais quadridimensionnelle, et permet l'expression simultanée de plusieurs idées.

Lorsque le francophone dit : " Demain, j'irai en Afrique à Dakar ", en mettant les mots dans cet ordre, le signeur va, quant à lui, signer sur la ligne du temps pour indiquer que l'action se déroulera " demain ", et faire le signe " en Afrique " et "à Dakar " en utilisant l'information redondante "espace selon le déroulement de l'action ". La LSF ne suit pas mot à mot le français (on parlerait alors de français signé gardant la syntaxe du français). La syntaxe du français est de type SVO (sujet-verbe-objet), celle de la LSF, de type OSV (objet-sujet-verbe). De plus, il n'existe pas de conjugaison en LSF. II suffit au signeur de situer l'action sur la "ligne du temps " (perpendiculaire à lui : derrière son épaule le passé, au niveau de son corps le présent et devant lui le futur). 
La LSF étant une langue sans territoire, on peut s'interroger sur ses modes de transmission, sachant que $10 \%$ des enfants sourds naissent de parents sourds et $90 \%$ de parents entendants. La LSF est transmise entre pairs dans les institutions spécialisées et au sein de la communauté sourde.

\section{Petit historique}

Jusqu'au XVIII' siècle, les sourds n'étaient pas jugés capables de communiquer par eux-mêmes. Pourtant, Saint Jérôme constatait déjà que les sourds " peuvent apprendre l'Évangile par les signes et utilisent dans la conversation journalière des mouvements expressifs de tout leur corps ". Au Xvie siècle, Montaigne écrivait : " Nos muets disputent, argumentent et content des histoires par signes. J'en ai vus de si souples et formés à cela qu'à la vérité, il ne leur manque rien à la perfection de se savoir faire entendre "(Essais, Livre II, ch. 12). C'est l'abbé de l'Épée (1712-1789) qui est à l'origine de la constitution de la communauté des sourds en France. À partir de l'observation de la gestuelle utilisée par deux jumelles sourdes-muettes, il établit qu'il existe un langage indépendant de l'expression orale. Décidé à rompre l'isolement qui est le lot des sourds-muets, il élabore une méthode d'enseignement et fonde un établissement à ses frais. C'est la première école des sourds, le berceau linguistique et culturel de la

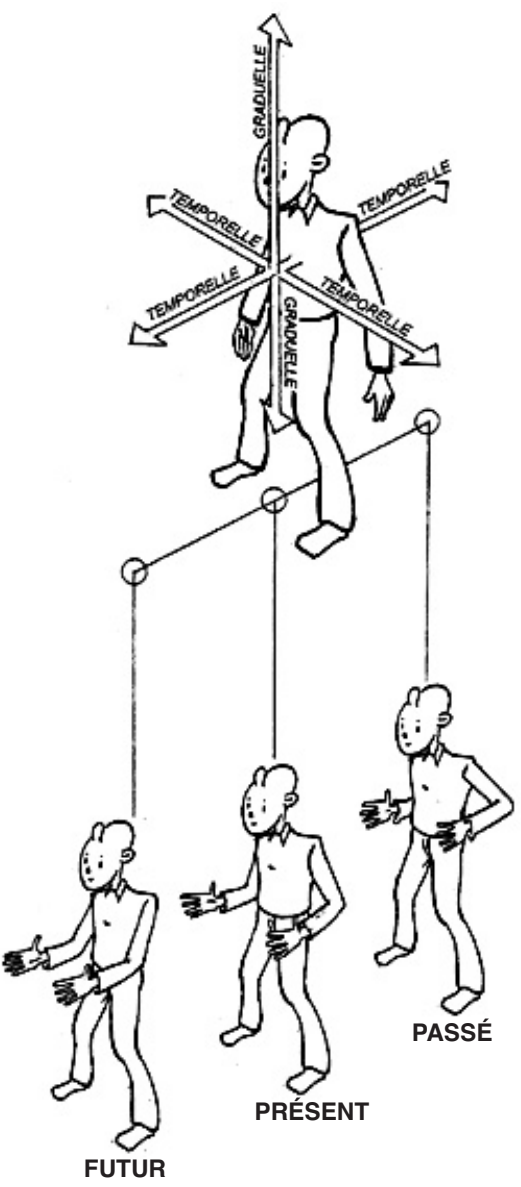

Illustration de L. Verlaine, dessinateur sourd, extraite de la présentation

de Mme Marie-Thérèse L'Huillier à la JMT 2008 communauté sourde. En 1774, il publie Institution des sourds-muets, puis en 1776 Institution des sourds-muets par la voie des signes méthodiques.

Le xixe siècle connaît une forte mobilisation de la communauté sourde. Malheureusement, des querelles d'éducateurs opposent les "gestualistes ", favorables à un enseignement gestuel issu de la culture des sourds et les "oralistes ", qui concentrent tous leurs efforts sur l'enseignement de la parole en rejetant la communication par signes, jugée "trop facile ". Les oralistes prennent bientôt le dessus et l'enseignement des signes est peu à peu supprimé en France. II est officiellement interdit en 1880, suite à un congrès international sur l'éducation des Sourds tenu à Milan. 
Cet interdit ne sera abrogé par le Ministère de la Santé qu'en 1977 et il faudra attendre 1991 (!) et la loi Fabius pour que l'Assemblée Nationale reconnaisse la liberté de choix entre la communication bilingue (langue des signes et français) et la communication orale dans l'éducation des jeunes sourds. Le résultat, souligné dans le rapport Gillot (du nom de la députée Dominique Gillot), est qu'en 1998, encore $80 \%$ des sourds sont illettrés. Suite à ce long interdit, des signes ont disparu et la langue, qui n'a pas évolué comme elle aurait pu le faire, présente de nombreuses lacunes lexicales.

Les années 1970-1980 sont marquées par l'action des sourds pour la défense de leurs droits citoyens, par la promotion de la LSF, le droit à l'éducation bilingue, la création de classes bilingues pour enfants sourds, la création de cours de LSF pour les entendants dans des associations, et la création officielle du métier d'interprète en LS (1980).

Avant 1975, la LSF était pratiquée presque exclusivement par la communauté sourde et les sourds étaient confrontés à toutes sortes d'obstacles dans leur vie quotidienne, n'ayant accès ni à l'information ni à la formation et ne disposant pas de moyen de communication. La situation s'améliore progressivement à partir de 1980 : la LSF devient accessible à un plus large public et les sourds commencent à avoir accès aux médias, aux études supérieures, aux nouvelles technologies, etc. De même, la recherche linguistique s'intéresse à la LSF.

\section{Évolution récente}

De gros bouleversements sont intervenus ces vingt dernières années, essentiellement sur trois plans : le développement des recherches linguistiques, la création de nouveaux signes et la place croissante de l'interprète.

Les recherches linguistiques doivent beaucoup à William Stokoe qui, dans les années 1960, fut le premier à étudier une langue des signes, l'American Sign Language (ASL). II la décrit en termes de phonèmes gestuels (gestèmes), qu'il nomme " chérèmes " (du grec kheir, la main). À la différence des phonèmes dont la segmentation dans le discours est séquentielle, les gestèmes se combinent spatialement et simultanément dans le signe. Stokoe en établit une transcription écrite et en 1965, publie un dictionnaire de l'ASL, avec Casterline et Croneberg.

En France, Christian Cuxac développe un modèle original qui aura une forte influence. II compare l'iconicité dans les langues vocalisées et les langues des signes et met en évidence la bifurcation entre deux pôles lors des activités discursives : la " grande iconicité "à visée illustrative (dire en montrant) et les signes standard, sans visée illustrative (dire sans montrer). Cuxac démontre que la grande iconicité fait partie des langues des signes, lesquelles disposent bien de DEUX manières de dire. Par conséquent, lorsqu'un signe " manque ", le concept peut être expliqué en grande iconicité, celle-ci constituant un procédé naturel de création de nouveaux signes standard. 
Ces recherches feront évoluer le regard des sourds sur leur propre langue qui s'en trouvera valorisée. La prise de conscience linguistique et métalinguistique chez les personnes sourdes débouchera sur un développement didactique et pédagogique de la LSF, enseignée en milieu scolaire et associatif.

La visée illustrative permet un dépassement des obstacles linguistiques et la création de nouveaux signes, indispensables au travail des interprètes qui facilitent l'accès des sourds à la modernité : accès aux médias (nécessité de créer des signes pour désigner les personnalités politiques), aux nouveaux métiers (nouvelles technologies, par exemple), à la culture (sciences, monuments,...), aux études supérieures dans toutes les disciplines, etc.

Le développement du métier d'interprète marque une évolution positive de la relation entre sourds et entendants. L'interprétation en langue des signes est parfaitement comparable à l'interprétation entre deux langues vocales (de l'anglais au français, de l'anglais au japonais, etc.). Cependant, la profession d'interprète LSF/français n'existe de façon organisée que depuis les années 1980. Auparavant, les échanges entre les sourds et leur entourage se faisaient de façon informelle grâce à la collaboration de leurs proches (enfants entendants de parents sourds ou amis entendants) ou d'intervenants dans le milieu de la surdité. L'interprète LSF/français est utile aux sourds comme aux entendants, puisqu'il pratique l'interprétation dans les deux sens.

C'est un secteur en plein essor, la demande d'intermédiaires entre sourds et entendants ne cessant d'augmenter. En effet, les sourds ont de plus en plus souvent recours à des interprètes en LSF : pour un entretien d'embauche, pour suivre une formation, comparaître devant un tribunal, etc.

Afin de répondre à ce besoin, des écoles de formation d'interprètes en langue des signes ouvrent leurs portes un peu partout, en France, en Europe et dans le monde entier. Les interprètes professionnels sont tous diplômés et soumis à un code déontologique qui s'appuie sur trois grands principes : la fidélité, la neutralité et le secret professionnel.

L'AFILS, Association française des interprètes en langue des signes, qui fête ses trente ans, regroupe les interprètes au sein d'une structure œuvrant à la promotion de la profession et à sa reconnaissance par les pouvoirs publics. Son répertoire comprend environ quatre-vingts professionnels, tous diplômés. Différentes universités assurent une formation d'interprète en langue des signes, notamment Paris 8 où enseigne Marie-Thérèse L'Huillier ou encore l'ESIT. Pour plus de détails, consulter le site de l'AFILS, www.afils.fr. 


\section{Bibliographie}

CUXAC, C. (2000). La langue des signes française, les voies de l'iconicité, Éditions Ophrys. CUXAC, C. (1996). Fonctions et structures de l'iconicité des langues des signes, Thèse de Doctorat d'État, Université Paris V.

CUXAC, C. (1985). Esquisse d'une typologie des langues des signes. In C. Cuxac (éd.), Autour de la Langue des Signes. Journées d'Études $n^{\circ}$ 10, UFR de Linguistique Générale et Appliquée, Université René Descartes.

STOKOE, W. C., CASTERLINE, D. \& CRONEBERG, C. G. (1965). A dictionary of ASL based on linguistic principles. Washington D. C., Gallaudet College Press.

STOKOE, W. C. (1960). Sign language structure : an outline of the visual communication systems of the American Deaf. Occasionnal Papers $n^{\circ} 8$, University of Buffalo.

http://ufr6.univ-paris8.fr/desshandi/supl/projets/site_lsf/culture histoire/histoire.php, site consulté le 10 septembre 2009

http://www.afils.fr/index2.htm, site consulté le 6 octobre 2009

http://www.websourd.org, site consulté le 6 octobre 2009, intervention de Marie-Thérèse L'Huillier à la 14e Université d'été 2008 sur le thème "Bilinguisme et société " 